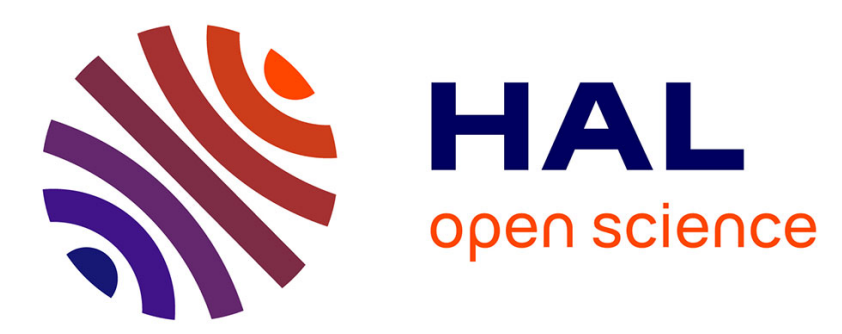

\title{
A DF-IBM/NSCD coupling framework to simulate immersed particle interactions
}

Talib Dbouk, Frédéric Perales, Fabrice Babik, Rémy Mozul

\section{To cite this version:}

Talib Dbouk, Frédéric Perales, Fabrice Babik, Rémy Mozul. A DF-IBM/NSCD coupling framework to simulate immersed particle interactions. Computer Methods in Applied Mechanics and Engineering, 2016, 309, pp.610-624. 10.1016/j.cma.2016.05.041 . hal-01364952

\section{HAL Id: hal-01364952 \\ https://hal.science/hal-01364952}

Submitted on 13 Sep 2016

HAL is a multi-disciplinary open access archive for the deposit and dissemination of scientific research documents, whether they are published or not. The documents may come from teaching and research institutions in France or abroad, or from public or private research centers.
L'archive ouverte pluridisciplinaire HAL, est destinée au dépôt et à la diffusion de documents scientifiques de niveau recherche, publiés ou non, émanant des établissements d'enseignement et de recherche français ou étrangers, des laboratoires publics ou privés. 


\title{
A DF-IBM/NSCD coupling framework to simulate immersed particle interactions
}

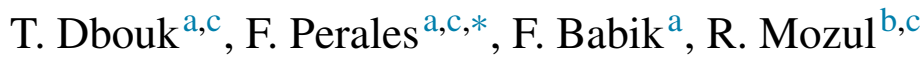 \\ ${ }^{a}$ Institut de radioprotection et de sûreté nucléaire (IRSN), PSN, CE Cadarache, BP3 - 13115 Saint-Paul-Lez-Durance, France \\ ${ }^{\mathrm{b}}$ Laboratoire de Mécanique et Génie Civil (LMGC) - UMR5508 - Université Montpellier II, CC048 Place Eugène Bataillon, 34095 Montpellier \\ cedex 5, France \\ ${ }^{\mathrm{c}}$ Laboratoire MIST, CNRS - IRSN - Université Montpellier II, France
}

\begin{abstract}
Immersed granular flows are present widely in different domains under different forms (at various scales) such as in nature (rivers, muds, atmosphere, blood...), and in many industrial applications (detergents, cosmetics, etc...). Studying such flows properly requires one to represent well the physics behind their dynamics: the fluid/solid interactions (FSI), the solid/solid interactions (SSI) and the coupling mechanisms at various scales.

In this work, a new coupling framework to simulate immersed granular flows has been developed. The FSI has been modeled using a direct-forcing immersed boundary method (DF-IBM) and implemented in the parallelized "PELICANS" C++ library. In this DF-IBM, all the mathematical equations, including the direct-forcing term, are discretized, both in space and time, and solved iteratively via a finite-volume and projection methods on Eulerian Grids. A sharp-edge interface, that can be smoothed, is used to represent the fluid/solid transition. The modeling of the multiple SSI at the grain's scale is based on the Non-Smooth Contact Dynamics (NSCD) approach developed in the "LMGC90" open-source library. The coupling of the two softwares "PELICANS" and "LMGC90", called Xper, provides an efficient framework to simulate and study dense immersed granular flows by taking into account, both advanced contact laws between grains, and hydrodynamic interactions.

We address in this paper the effects of imposing a fluid-ring numerically (or fluid-mesh-cells) around two settling solid disks on modifying their dynamics. The DF-IBM approach implemented in Xper is validated, on a 2D flow over a stationary rigid cylinder benchmark, and on the settling of a rigid buoyant sphere in an incompressible laminar fluid at different Reynolds numbers. The numerical results are in good agreement with experimental and numerical data from the literature.
\end{abstract}

$\mathrm{c}$

Keywords: Immersed granular flows; Fluid/structure interaction; Direct-forcing; Immersed boundary method; Non-smooth contact dynamics

\section{Introduction}

Sands of a sea, rocks of a river, dust particles of an atmosphere, red cells of a blood, solid particles of cosmetic and detergent products are all some examples on rigid grains or particles of a high concentration immersed in a

\footnotetext{
* Corresponding author at: Institut de radioprotection et de sûreté nucléaire (IRSN), PSN, CE Cadarache, BP3 - 13115 Saint-Paul-Lez-Durance, France.

E-mail address: frederic.perales@irsn.fr (F. Perales).
} 
fluid. Whenever these immersed granular particles are subject to a flow in motion, interactions arise between the particles themselves, called Solid/Solid interactions (SSI) and also between the particles and the surrounding fluid, called Fluid/Solid Interactions (FSI). Studying such flows experimentally is extremely important to understand and characterize their rheology. However, in many situations, the experimental setups may be a difficult task and can be replaced or completed by numerical investigations.

Experimentally speaking, many scientists performed a lot of measurements over the years in an attempt to understand the rheology behind such flows. For example, some researchers [1] measured the settling velocity of a rigid sphere in a viscous fluid. Others conducted rheometry measurements for the flows of highly concentrated nonBrownian suspensions of hard spheres. N. Blaise [2] characterized the wavefront dynamics in a dam break problem for a hyperconcentrated suspension for a wide variety of particle concentrations. Recently, F. Blanc et al. [3] measured the fall velocity of a dense ball in an oscillatory cross-sheared concentrated suspension.

Numerically speaking, many methods have been developed successfully over the years to account for the presence of rigid bodies in a fluid as we will see in the coming section. These methods modeled immersed granular flows at different scales: microscopic, mesoscopic and macroscopic. However, the choice of a method is a major challenge and depends on many factors such as the computational time needed. The microscopic modeling approach is the most costly one with an extreme computational effort but the most complete one from a modeling point of view. In fact, there are many lacks in the mesoscopic and macroscopic approaches for predicting rheological behavior of the flow such as the highly concentrated suspensions where the microstructure plays a vital role. For these reasons, an objective for us is the numerical modeling on a microscopic scale in an attempt to constitute later macroscopic rheology laws. In this paper, for the first time to our knowledge, we show with numerical examples the effect of imposing/non-imposing a numerical fluid-ring (or fluid-mesh-cells) around a particle on the dynamics of two settling particles in a vertical channel. Most studies follow same or similar numerical strategies in modeling the contact laws between the particles, but without any numerical tests, illustration or validations on the effect for example of this imposing/non-imposing of a numerical fluid-ring around the particles on their dynamics. We show in our work that it has a great effect on the dynamics at the microscopic scale, which might thus regenerate larger kinematics modifications in the macroscopic behavior of an immersed granular flow.

The present paper is organized as follows. The numerical fluid-structure interactions modeling and the validation are presented in Section 2. Section 3 presents numerical coupling framework and its associated benchmark. The effects of the forcing/not-forcing of many methods have been developed around two settling disks in contact on the dynamics are investigated in Section 4.

\section{The fluid-structure interactions modeling}

\subsection{The direct-forcing-immersed boundary method}

In the present study, the Direct-Forcing Immersed Boundary Method (DF-IBM) is the numerical method used to account for the presence of rigid bodies immersed in a fluid (Fig. 1). The flow field is solved on an Eulerian grid discretization that does not need to conform to the solid boundaries. The solids are discretized on a Cartesian grid and their boundaries are represented by a set of Lagrangian points.

The original Immersed Boundary Method (IBM) goes back to the early works of Peskin [4-6] and many others who added their finger-prints like [7,8]. The history of the IBM is not the main task of this work and thus we leave it to many authors in literature who tackled it such as in [9]. Briefly speaking, the DF-IBM is defined by modeling the fluid with the classical Navier-Stokes equations and then by imposing a velocity penalization term and a velocity time integral in the momentum equation only at mesh points that may define a material point of an immersed object. The general idea is to penalize and force-directly a no-slip boundary condition (BC) on the object's surface surrounded by the fluid. This methodology permits fast and efficient numerical approximations on Cartesian Eulerian Grids and may avoid body-fitted unstructured meshes and costly remeshing procedure.

The incompressible fluid dynamics, including an immersed rigid body (Fig. 1) of volume $\Omega_{s}$ and boundary $\partial \Omega_{s}$, are governed by the transient directly-forced Navier-Stokes equations (1)-(2):

$$
(1-\eta) \rho_{f} \frac{\partial \mathbf{u}}{\partial t}+(1-\eta)\left[\rho_{f} \mathbf{u} \cdot \nabla \mathbf{u}-\nabla \cdot \boldsymbol{\sigma}-f_{e x t}\right]=\rho_{f} \eta \frac{\left(\mathbf{u}_{\mathbf{s}}-\mathbf{u}\right)}{\Delta t}
$$




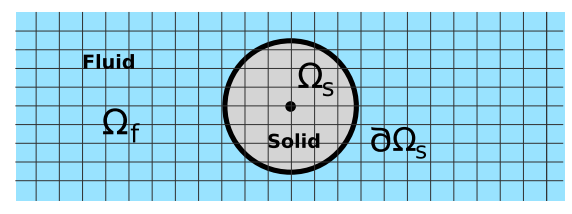

Fig. 1. An immersed boundary.

and

$$
\nabla \cdot \mathbf{u}=0
$$

where $\sigma$ is the fluid stress tensor defined as:

$$
\boldsymbol{\sigma}=-p \mathbf{I}+\boldsymbol{\tau} \quad \boldsymbol{\tau}=\mu\left[\nabla \mathbf{u}+(\nabla \mathbf{u})^{T}\right]
$$

and $f_{e x t}$ an external applied force field vector (such as the gravitational force), $\mathbf{u}$ the fluid velocity vector, $\mathbf{u}_{\mathbf{s}}$ the immersed rigid body velocity, $\rho_{f}$ the fluid density, $p$ the pressure, $\mu$ the fluid dynamic viscosity, and $\eta$ is a sharpedged coefficient defined as the following:

$$
\begin{array}{ll}
\eta=1-\epsilon & \forall \overrightarrow{\mathbf{X}}(\mathbf{x}, \mathbf{y}, \mathbf{z}) \in \Omega_{\mathbf{s}} \\
\eta=\epsilon & \forall \overrightarrow{\mathbf{X}}(\mathbf{x}, \mathbf{y}, \mathbf{z}) \in \Omega_{\mathbf{f}}
\end{array}
$$

with $\epsilon$ a parameter such as $\epsilon \ll 1$.

The boundary conditions and the initial conditions are respectively: $\mathbf{u}=\mathbf{u}_{\text {boundary }}$ on the boundary of $\Omega_{f}$ and $\mathbf{u}\left(t_{0}\right)=\mathbf{u}_{0}$ inside $\Omega_{f}$ at the initial time $t=t_{0}$. Note that this formulation can be viewed as a penalization method.

In this work $\epsilon$ is taken equal to $10^{-12}$. However, the effect of the parameter $\epsilon$ on the accuracy of this method is minor as explained in literature by [8]. The authors have established rigorous estimates of the error induced by such penalization methods and showed numerically their efficiency. Note that if $\eta=1$, the Eq. (1) reduces to $\mathbf{u}=\mathbf{u}_{\mathbf{s}}$ which is a direct forcing of the fluid velocity field on and inside the immersed rigid boundary. Note that the shape of the function $\eta$ is not the issue of this work and the form of this coefficient is left for investigation in a further work.

The Navier-Stokes equations (1)-(2) are discretized temporally using an Explicit Euler Scheme and spatially via a Marker-And-Cell scheme [10] on staggered grids. The numerical system is solved iteratively by a Pressure Schur Complement method (PSC) inherited from the classical Discrete Projection Methods provided by Chorin [11] and Van Kan [12]. We will describe the method briefly, however a detailed description of the whole numerical technique can be found in [13].

A semi-discretization of Eqs. (1)-(2) in a semi-implicit manner with respect to time results in:

$$
\begin{aligned}
& \left(1-\eta^{n}\right) \rho_{f} \frac{\partial \mathbf{u}^{n+1}}{\partial t}+\left(1-\eta^{n}\right) \rho_{f}\left(\mathbf{u}^{n} \cdot \nabla\right) \mathbf{u}^{n+1} \\
& \quad=\left(1-\eta^{n}\right)\left(-\nabla p^{n}+\nabla \cdot \boldsymbol{\tau}\left(\mathbf{u}^{n+1}\right)+f_{\text {ext }}^{n}\right)+\rho_{f} \eta^{n} \frac{\left(\mathbf{u}_{s}^{n+1}-\mathbf{u}^{n+1}\right)}{\Delta t_{f}} \\
& \nabla \cdot \mathbf{u}^{n+1}=0
\end{aligned}
$$

where $\Delta t_{f}$ is the fluid time step, superscript $n$ the quantities at a time $t_{n}, n+1$ the quantities at time $t_{n+1}, p^{n+1}$ the fluid pressure, $\mathbf{u}^{n+1}$ the velocity approximations. Note that the fluid pressure and the velocity approximations have to be corrected later in order to satisfy the Eq. (7).

The term $\partial \mathbf{u}^{n+1} / \partial t$ is an approximate of the temporal velocity derivative which depends on order scheme choice. The projection method is described in four fractional step scheme as follows:

1. Knowing, $\eta^{n}, p^{n}$ and $\mathbf{u}^{n}$, find an intermediate fluid velocity field $\tilde{\mathbf{u}}^{n+1}$ such that:

$$
\begin{aligned}
& \left(1-\eta^{n}\right) \rho_{f} \frac{\tilde{\mathbf{u}}^{n+1}-\mathbf{u}^{n}}{\Delta t}+\left(1-\eta^{n}\right) \rho_{f}\left(\mathbf{u}^{n} \cdot \nabla\right) \tilde{\mathbf{u}}^{n+1} \\
& \quad=\left(1-\eta^{n}\right)\left(-\nabla p^{n}+\nabla \cdot \boldsymbol{\tau}\left(\tilde{\mathbf{u}}^{n+1}\right)+f_{e x t}^{n}\right)+\rho_{f} \eta^{n} \frac{\left(\mathbf{u}_{\mathbf{s}}^{n+1}-\tilde{\mathbf{u}}^{n+1}\right)}{\Delta t_{f}} .
\end{aligned}
$$


2. Find $\varphi$ such that:

$$
\Delta \varphi=\frac{\rho_{f}}{\Delta t_{f}} \nabla \cdot \tilde{\mathbf{u}}^{n+1} .
$$

3. Correct the fluid velocity with the equation:

$$
\mathbf{u}^{n+1}=\tilde{\mathbf{u}}^{\mathbf{n}+\mathbf{1}}-\frac{\Delta t_{f}}{\rho_{f}} \nabla \varphi .
$$

4. Correct the fluid pressure such that:

$$
p^{n+1}=p^{n}+\varphi \text {. }
$$

\subsection{The drag and lift forces acting on an immersed rigid body}

The total fluid force, denoted $\mathbf{F}_{F S I}$, applied on the immersed boundary of a rigid body is defined as:

$$
\mathbf{F}_{F S I}=\int_{\partial \Omega_{S}} \boldsymbol{\sigma} \cdot \mathbf{n} d A
$$

where $\mathbf{n}$ denotes the unit normal vector to the surface $\partial \Omega_{s}$.

Dividing Eq. (1) by $(1-\eta)$ and with no external forces, we obtain:

$$
\rho_{f} \frac{\partial \mathbf{u}}{\partial t}+\left[\rho_{f} \mathbf{u} \cdot \nabla \mathbf{u}-\nabla \cdot \boldsymbol{\sigma}\right]=\rho_{f} \frac{(1-\epsilon)}{\epsilon} \frac{\mathbf{u}_{\mathbf{s}}-\mathbf{u}}{\Delta t_{f}} .
$$

In the rigid body, the first and the second terms to the left of Eq. (13) become negligible, which leads to the following:

$$
\left[\nabla \cdot \boldsymbol{\sigma}=\rho_{f} \frac{(1-\epsilon)}{\epsilon} \frac{\mathbf{u}_{\mathbf{s}}-\mathbf{u}}{\Delta t_{f}}\right]_{\forall \overrightarrow{\mathbf{X}}(x, y, z) \in \Omega_{s}} .
$$

Integrating Eq. (14) over the domain of the body gives:

$$
\int_{\Omega_{s}} \nabla \cdot \sigma d \Omega=\int_{\Omega_{s}} \rho_{f} \frac{(1-\epsilon)}{\epsilon} \frac{\mathbf{u}_{\mathbf{s}}-\mathbf{u}}{\Delta t_{f}} d \Omega .
$$

Now, applying the Gauss-Ostrogradsky divergence theorem for the term to the left of Eq. (15) and using the definition of the force in Eq. (12) lead to the following:

$$
\int_{\Omega_{s}} \nabla \cdot \boldsymbol{\sigma} d \Omega=\int_{\partial \Omega_{s}} \boldsymbol{\sigma} \cdot \mathbf{n} d A=\mathbf{F}_{F S I}=\int_{\Omega_{s}} \rho_{f} \frac{(1-\epsilon)}{\epsilon} \frac{\mathbf{u}_{\mathbf{s}}-\mathbf{u}}{\Delta t_{f}} d \Omega .
$$

Eq. (16) permits to compute numerically the total fluid force applied on an immersed rigid body of volume $\Omega_{s}$. This method is denoted here as a Penalization Term Integration Technique (PTIT). In fact, it is based on the definition of the coefficient $\eta$ as a function of $\epsilon$ in Eq. (5) and on previous works in literature on generalized Brinkman type Models (porous medium with variable permeability) [14,15]. Note that in this PTIT technique, we do not impose any empirical formulations like the ones that can be used for example for writing the drag coefficient as function of Reynolds number. Some authors in the literature [16] implied empirical formulations for the drag coefficient to handle immersed particulate flows at various flow regimes. However, this limits the solver to handle only some predefined shapes for the immersed bodies (i.e. spheres, squares, cylinders) because the drag coefficient laws for such simple shapes had been well constituted in the literature for general flows at different Reynolds numbers.

The drag and lift forces are defined, respectively, as the following (Fig. 2):

$$
F_{D}=\left(-\left(\mathbf{F}_{F S I}\right)_{x}, 0,0\right) ; \quad F_{L}=\left(0,-\left(\mathbf{F}_{F S I}\right)_{y}, 0\right) .
$$




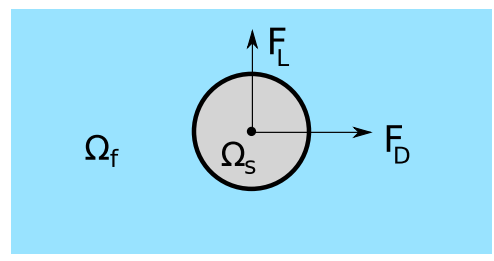

Fig. 2. Drag and lift forces acting on an immersed cylinder $\Omega_{S}$.

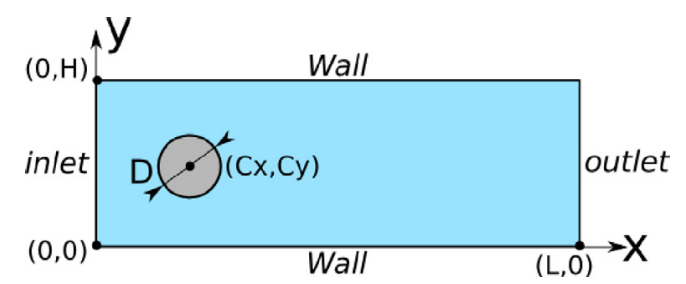

Fig. 3. 2D flow over a stationary cylinder.

Table 1

2D flow over a stationary cylinder: parameters at $R e=20$.

\begin{tabular}{lllll}
\hline Uniform mesh & $D / \Delta_{x}$ & $\Delta t_{f}$ & $\mu$ & $\rho_{f}$ \\
\hline$\Delta_{x}=\Delta_{y}=0.01$ & 10 & $0.01 \mathrm{~s}$ & $0.001 \mathrm{~Pa} \mathrm{~s}$ & $1 \mathrm{~kg} / \mathrm{m}^{3}$ \\
\hline
\end{tabular}

The drag $C_{D}$ and lift $C_{L}$ coefficients of the forces acting on a cylinder of diameter $D$ are defined as:

$$
C_{D}=\frac{F_{D}}{\frac{1}{2} \rho_{f} \bar{u}^{2} D} ; \quad C_{L}=\frac{F_{L}}{\frac{1}{2} \rho_{f} \bar{u}^{2} D}
$$

where $\bar{u}$ is the characteristic velocity arising from the definition of Reynolds number $R e$ at the scale of a cylinder:

$$
R e=\frac{\rho_{f} \bar{u} D}{\mu} .
$$

The DF-IBM (Eqs. (1)-(2)) and the drag and lift computations are implemented inside the parallelized PELICANS code as a new solver [17].

\subsection{Validation on a stationary immersed rigid body}

In what follows, the numerical framework and its implementation are validated on the transient incompressible 2D flow over a stationary cylinder for two Reynolds numbers, 20 and 100. The numerical results obtained are compared to the Test case $2 \mathrm{D}-1(R e=20$ at $\bar{u}=0.2 \mathrm{~m} / \mathrm{s})$ and Test case $2 \mathrm{D}-2(R e=100$ at $\bar{u}=1 \mathrm{~m} / \mathrm{s})$ in the benchmark results provided by [18].

Fig. 3 shows the geometry being used with respective dimensions: $(\mathrm{Cx}=\mathrm{Cy}=0.2 \mathrm{~m} ; \mathrm{D}=0.1 ; \mathrm{H}=0.41 \mathrm{~m}$ and $\mathrm{L}=2.2 \mathrm{~m}$ ). The same boundary conditions (BC) in [18] were imposed: a homogeneous Dirichlet condition on the walls, a Dirichlet velocity field on the inlet (with a parabolic profile) and a Neumann BC on the outlet.

\subsubsection{D flow over a stationary cylinder at $R e=20$}

Table 1 shows the parameters for $R e=20$.

Fig. 4(a)-(d) shows the velocity field, the pressure field and their contours at $R e=20$. After $10 \mathrm{~s}$, the flow is already in a steady state. The Fig. 4(e)-(f) shows the drag and lift coefficients computed along the 10 s. The Table 2 presents the comparison between the reference values provided by [18] and the results.

The obtained values at $D / \Delta_{x}=10$ for the pressure-difference $\Delta p=\left(p\left(C_{x}-\frac{D}{2}, C_{y}\right)-p\left(C_{x}+\frac{D}{2}, C_{y}\right)\right)$ and drag coefficient are in good agreement with the benchmark values provided by [18]. However, a slight difference in 

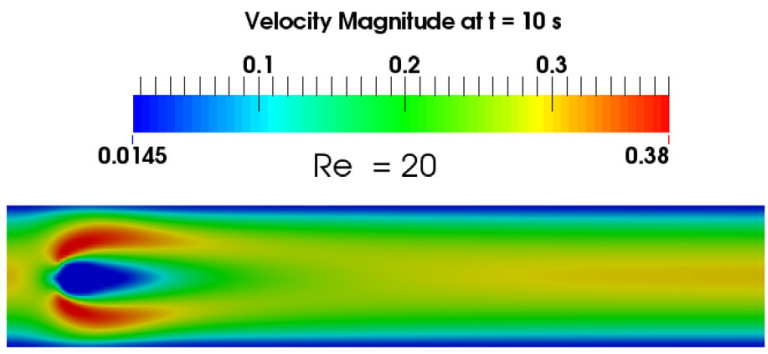

(a) velocity field.

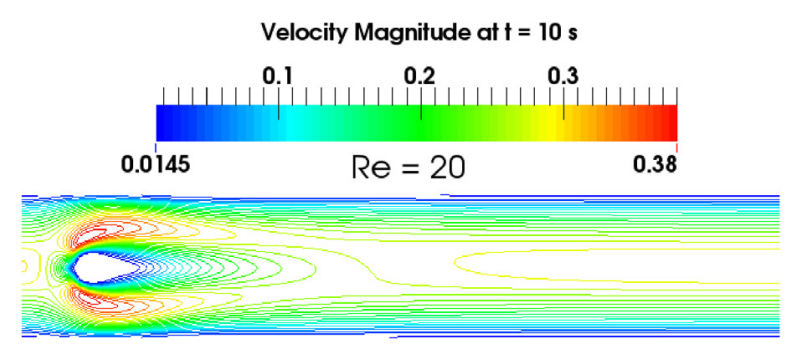

(c) velocity contours.

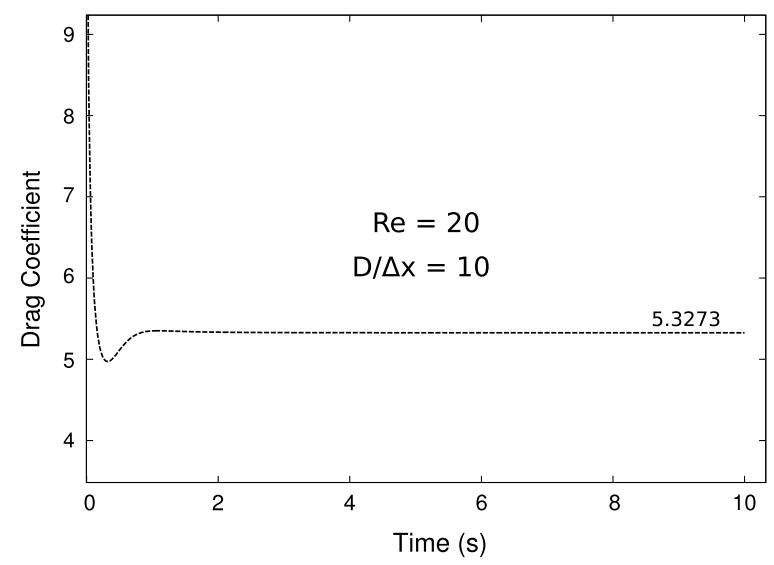

(e) Drag coefficient.

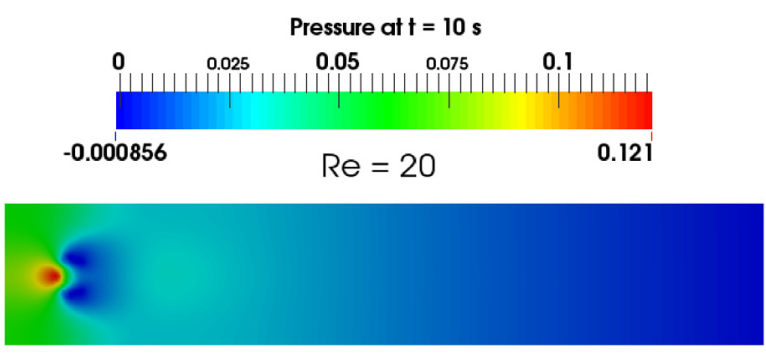

(b) pressure field.

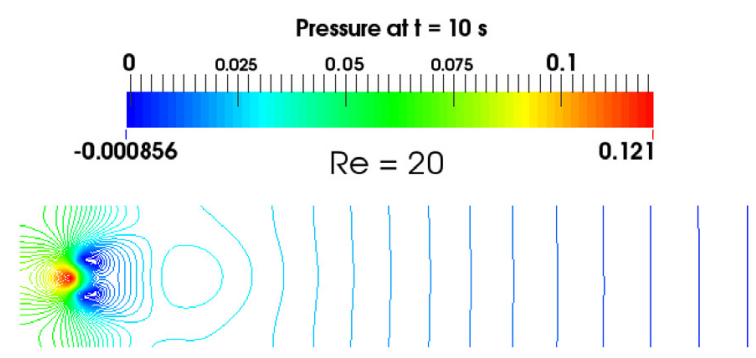

(d) pressure contours.

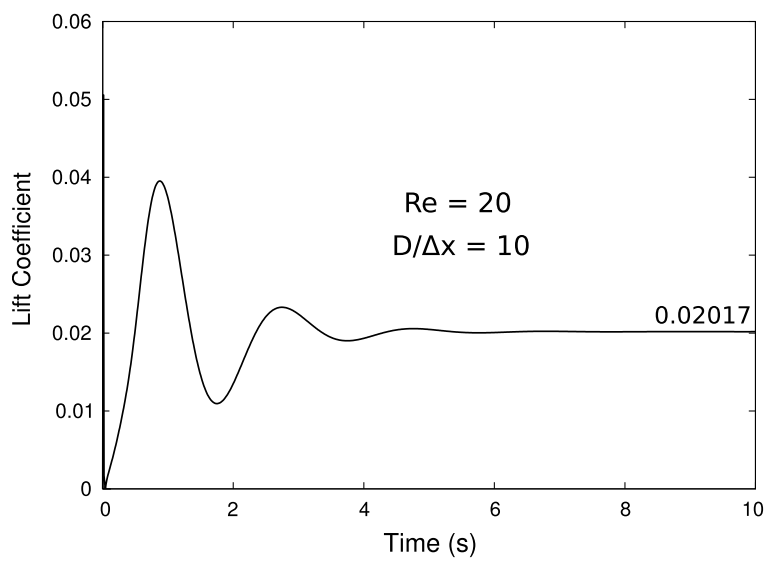

(f) Lift coefficient.

Fig. 4. 2D flow over a stationary cylinder at $R e=20$ and $D / \Delta_{x}=10$.

Table 2

Steady values for pressure-difference, drag and lift coefficients at $R e=20$.

\begin{tabular}{lll}
\hline & Reference values [18] & Present solver $\left(D / \Delta_{x}=10\right)$ \\
\hline $\mathbf{C}_{D}$ & {$[5.57: 5.59]$} & 5.3273 \\
$\mathbf{C}_{L}$ & {$[0.0104: 0.0110]$} & 0.02017 \\
$\Delta p$ & {$[0.1172: 0.1176]$} & 0.10570
\end{tabular}

the drag is observed and an over estimated value is obtained for the lift coefficient. This may be due to the different schemes (spatial or temporal), different methods being used in different solvers and to the sensitivity of the numerical results on the diameter-to-space-step (DSS) ratio $D / \Delta_{x}$ as it is shown in the next section. 


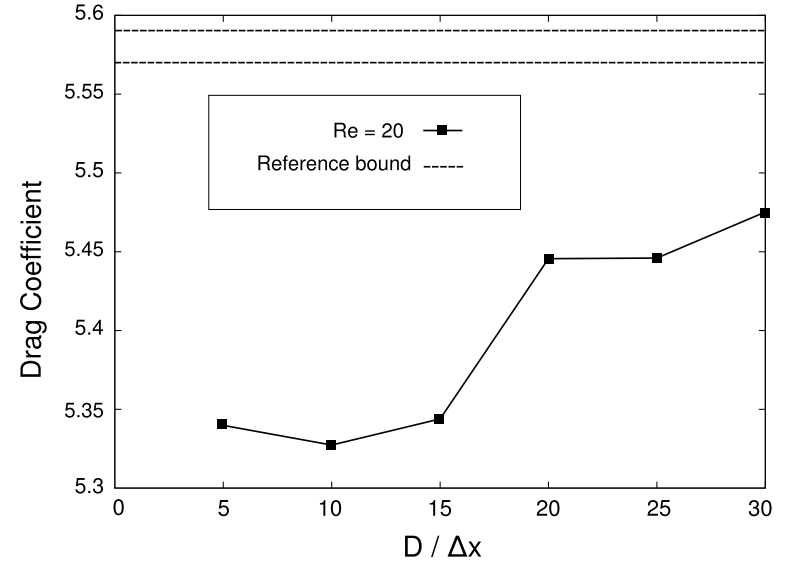

(a) Drag coefficient.

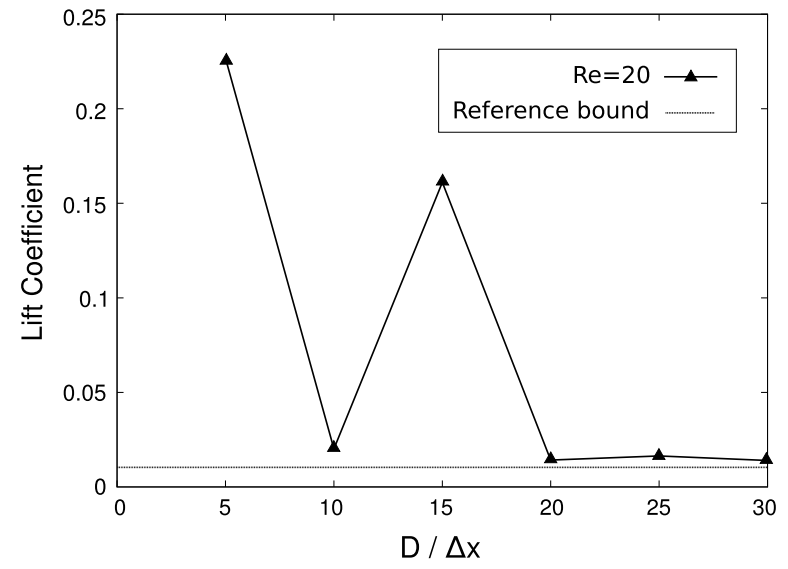

(b) Lift coefficient.

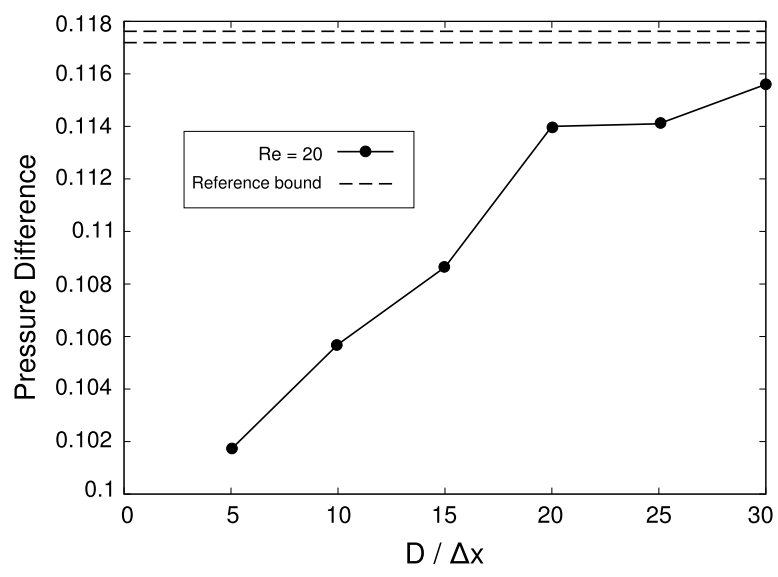

(c) Pressure-difference.

Fig. 5. Drag, lift and pressure-difference coefficients versus the DSS ratio.

\subsubsection{Effect of the diameter-to-space-step (DSS) ratio $D / \Delta_{x}$}

The sensitivity of the pressure-difference, drag and lift coefficients computations to six different values of the DSS ratio $D / \Delta_{x}=[5: 30]$ is studied. We emphasize that, whenever the $D / \Delta_{x}$ is increased, the time step $\Delta t_{f}$ is reduced and fixed in the solver to ensure a numerical stability with a 0.2 Courant-Friedrichs-Lewy (CFL) coefficient fulfilling the condition: $\mathbf{C F L}<1$. We recall that the mesh is always uniform with $\Delta_{y}=\Delta_{x}$. The Figs. 5 and 6 shows clearly the effect of the DSS ratio on the numerical results and the convergence is achieved at 20. The values in Table 3, as expected, show that our computed pressure-difference, drag and lift coefficients values approach the reference benchmark ones [18] as the DSS ratio increases.

\subsection{3. $2 D$ flow over a stationary cylinder at $R e=100$}

Fig. 7(a)-(d) shows the velocity field, the pressure field and their contours at $R e=100$. We can see that the symmetry in the flow is broken and vortices are generated behind the wake region of the immersed cylinder which is an expected phenomenon for flows at moderate to high Reynolds numbers. The Fig. 7(e)-(f) shows the drag and lift coefficient versus time at $R e=100$. Oscillations are observed due to the vortex generations behind the wake of cylinder. For the benchmark, the maximum values for both drag and lift coefficients are requested. The values are shown in Table 4. By increasing the DSS ratio, our results become close to the benchmark results obtained by [18]. 


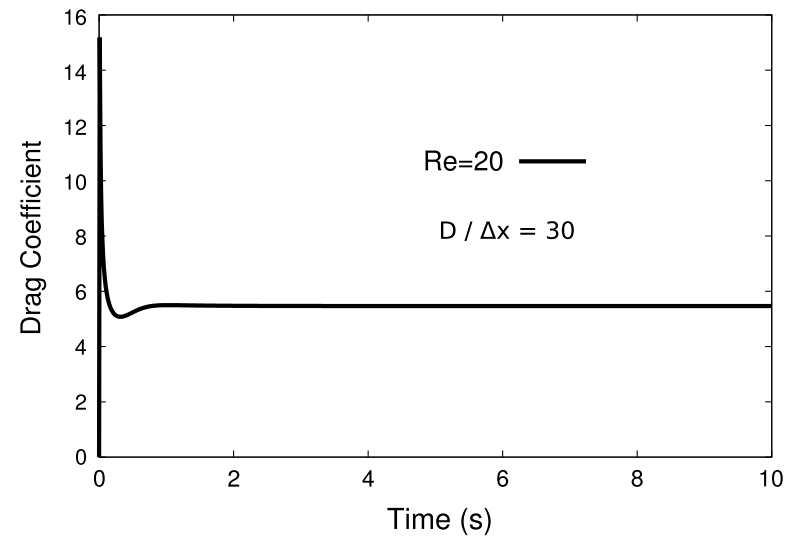

(a) Transient drag.

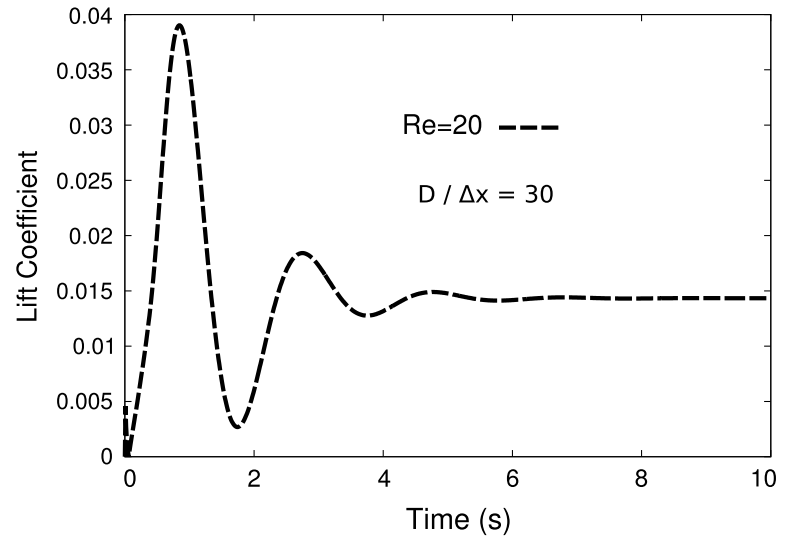

(b) Transient lift.

Fig. 6. Transient drag and lift coefficients at $\mathbf{D S S}=30$.

Table 3

Values for pressure-difference, drag and lift coefficients at $R e=20$ and $t=10 \mathrm{~s}$.

\begin{tabular}{llll}
\hline Present solver $D / \Delta_{x}$ & $C_{D}$ & $C_{L}$ & $\Delta p$ \\
\hline 5 & 5.3398 & 0.2267 & 0.1017 \\
10 & 5.3273 & 0.02017 & 0.10570 \\
15 & 5.3439 & 0.1611 & 0.10864 \\
20 & 5.4456 & 0.0142 & 0.1140 \\
25 & 5.4460 & 0.0164 & 0.1141 \\
30 & 5.4752 & 0.0140 & 0.1156 \\
Reference values [18] & {$[5.57: 5.59]$} & {$[0.0104: 0.0110]$} & {$[0.1172: 0.1176]$} \\
Range variation [18] & {$[5.50: 5.81]$} & {$[0.0104: 0.0329]$} & {$[0.1054: 0.1230]$} \\
\hline
\end{tabular}

Table 4

Values for pressure-difference, drag and lift coefficients at $R e=100$ and $t=50 \mathrm{~s}$.

\begin{tabular}{llll}
\hline Present solver $D / \Delta_{x}$ & $C_{D}$ & $C_{L}$ & $\Delta p$ \\
\hline 5 & 2.97 & 0.574 & 2.0959 \\
10 & 2.788 & 0.441 & 2.2158 \\
15 & 2.911 & 0.9316 & 2.3128 \\
20 & 2.980 & 0.715 & 2.3438 \\
25 & 2.976 & 0.720 & 2.3537 \\
30 & 3.076 & 0.920 & 2.3710 \\
Reference values [18] & {$[3.22: 3.24]$} & {$[0.99: 1.010]$} & {$[2.46: 2.50]$} \\
Range variation [18] & {$[3.08: 4.12]$} & {$[0.72: 1.61]$} & {$[2.13: 3.14]$} \\
\hline
\end{tabular}

\section{Immersed rigid body dynamics}

\subsection{The numerical framework}

There are several numerical approaches in the literature to account for friction or contact mechanics modeling between the particles. For detailed analyses of such contact mechanics modeling involving friction laws, we mention the early works of [19,20], and [21]. However, our developed FSI solver implemented inside the PELICANS software is coupled to the contact mechanics SSI solver called LMGC90 [22]. The SSI solver LMGC90 relies on the Non Smooth Contact Dynamics approach of $[21,23]$ where it solves for rigid bodies motion (Newton's second law) taking into account the multiple solid to solid interactions based on rigid friction laws. An advantage in this contact mechanics solver is that one can treat polydisperse cases, with total particle/particle contacts-solvings thanks to the NSCD 

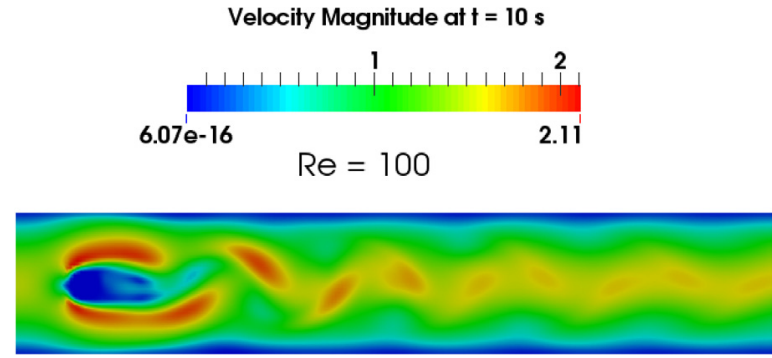

(a) Velocity field.
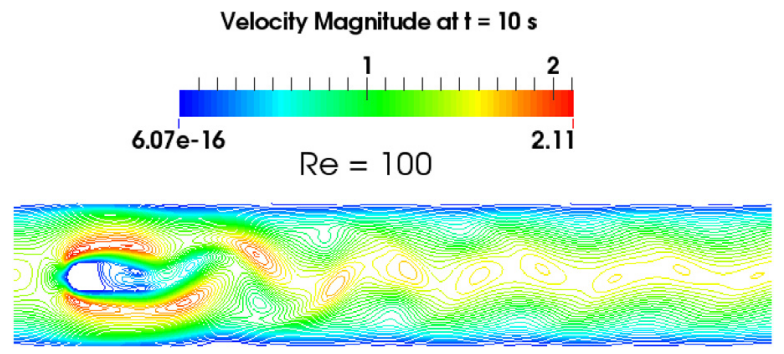

(c) Velocity contour.

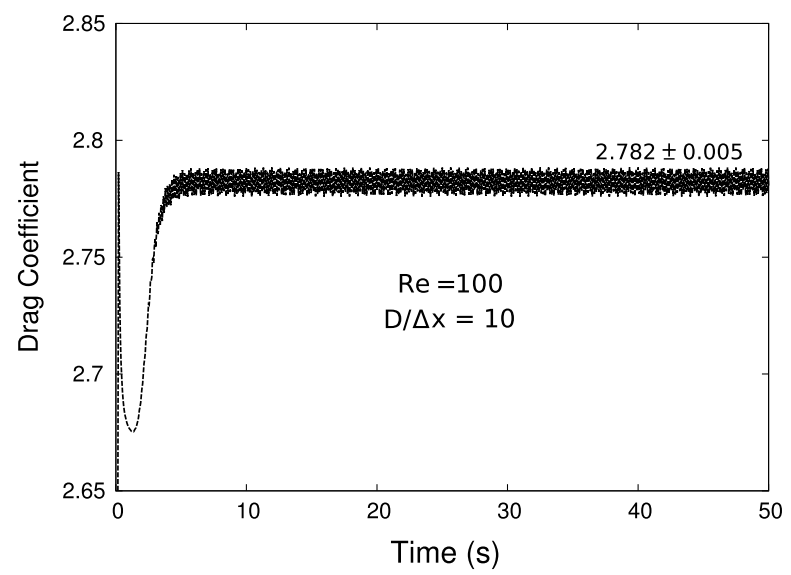

(e) Drag coefficient.
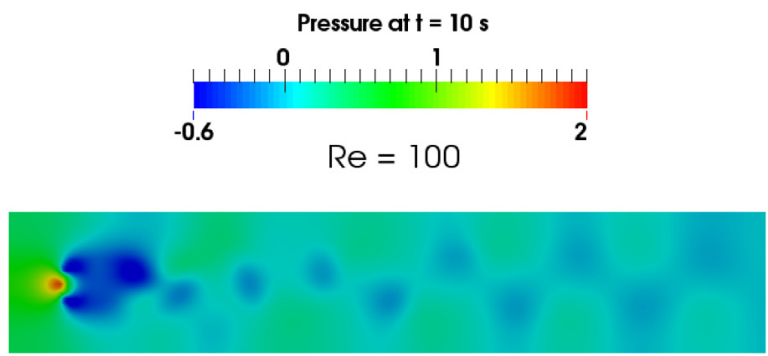

(b) Pressure field.
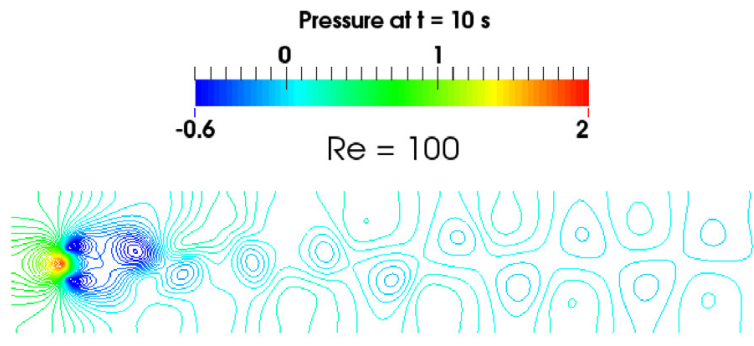

(d) Pressure contour.

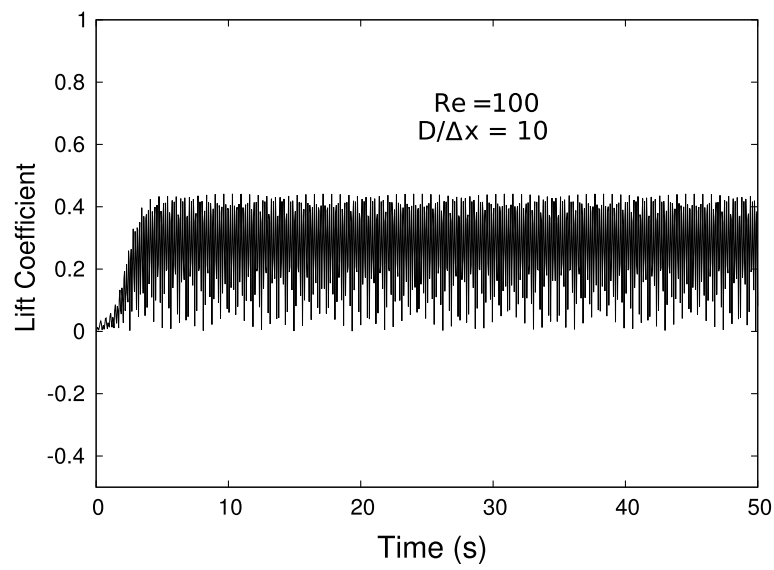

(f) Lift coefficient.

Fig. 7. 2D flow over a stationary cylinder at $R e=100$ and $D / \Delta_{x}=10$.

contact/friction laws and the powerful numerical algorithms already implemented in this LMGC90 package. We should mention that the LMGC90 [22] software has been developed over the last 25 years and was very well validated numerically and experimentally on many dry granular media dynamics applications.

As we mentioned above, the particles polydispersity in LMGC90 is advantageous for further future developments. In fact, in many previous studies (if not most), for describing the contact laws between the particles, many authors assume that the particles are spherical restricted and remain spherical even after impacts [24,25], which limits further possibilities of applying the method to a wide variety of applications.

The dynamical system in our work of immersed bodies in a fluid is given by the following:

$$
\begin{aligned}
\mathbf{F}_{T} & =m_{b} \frac{\partial \mathbf{u}_{b}}{\partial t} & \mathbf{T}_{T} & =\mathbf{I}_{b} \frac{\partial \omega_{b}}{\partial t} \\
\mathbf{F}_{T} & =\mathbf{F}_{F S I}+\mathbf{F}_{C}+m_{b} \mathbf{g} & \mathbf{T}_{T} & =\mathbf{T}_{F S I}+\mathbf{T}_{C}
\end{aligned}
$$




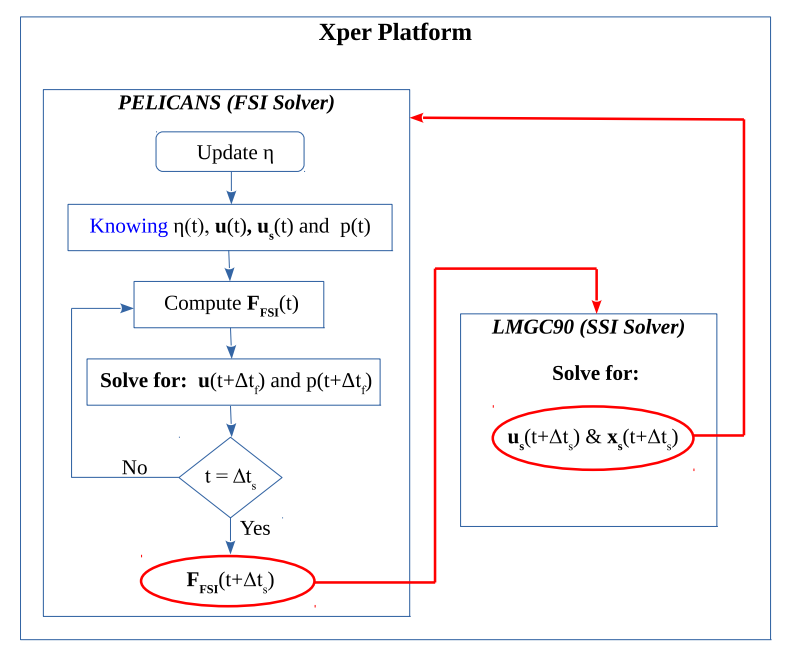

Fig. 8. Numerical algorithm implemented in the Xper code.

where $\mathbf{F}_{T}$ and $\mathbf{T}_{T}$ are the total forces and torques respectively acting on an immersed body, $m_{b}$ the mass, $\mathbf{u}_{s}=\frac{\partial \mathbf{x}_{s}}{\partial t}$ and $\omega_{b}$ the linear and angular velocities respectively, $\mathbf{g}$ the gravitational acceleration, $\mathbf{I}_{b}$ the immersed body inertia matrix, $\mathbf{F}_{C}$ and $\mathbf{T}_{C}$ the SSI forces and torques respectively, $\mathbf{F}_{F S I}$ the FSI force (Eq. (16)), and $\mathbf{T}_{F S I}=\mathbf{r} \times \mathbf{F}_{F S I}$ the FSI torque with $\mathbf{r}$ being the local position relative to the immersed body centroid. The local velocity, on the Eulerian grid, in the immersed body (the right term of Eq. (1)) is defined by $\mathbf{u}_{s}=\mathbf{u}_{b}+\omega \times \mathbf{r}$.

The integration of the system (20) relies on a Leap-frog three-time-level scheme. Note that the solid time step is defined by $\Delta t_{s}=\beta \Delta t_{f}$ where $\beta \geq 1$ is a relaxation coefficient accounting for the weak coupling between the two FSI and SSI solvers and their convergence.

\subsection{The numerical platform}

Fig. 8 shows the numerical algorithm of the Xper platform. At a time t, upon knowing the different fields: the state $\eta$, the fluid velocity, the solid velocity and the pressure, the $\mathbf{F}_{F S I}$ force is computed in PELICANS. Then, the new fluid velocity, the pressure fields and the new $\mathbf{F}_{F S I}$ force are all computed for a next time step $t+\Delta t_{f}$. The sequence is repeated until the solid time $t+\Delta t_{s}$ is reached. Now, the latest $\mathbf{F}_{F S I}$ is passed to the LMGC90 solver to solve the dynamics of the immersed solid by computing a new solid velocity including the effects of any contacts with other immersed solids. The latest solid velocity is then passed again to the solver PELICANS.

One of the great advantages in Xper platform is the parallelism. This decreases effectively the computation time needed to conduct a simulation, especially when treating later thousands of immersed bodies in a fluid.

\subsection{Validation on the $3 D$ settling of a sphere}

In what follows, the coupling and the associated software, Xper, are validated on the 3D simulations of a rigid sphere. The results are compared to the experiments of Ten Cate et al. [1].

The domain is rectangular with $L_{x}=L_{y}=10 \mathrm{~cm}$ and $L_{z}=16 \mathrm{~cm}$ and the diameter of the rigid sphere is $D=1.5 \mathrm{~cm}$. The density of the sphere is $\rho_{s}=1120 \mathrm{~kg} / \mathrm{m}^{3}$. For the fluid, four Reynolds number $R e=1.5,4.1,11.6$ and 32.3 are considered. The associated parameters are presented in Table 5. The time step respects the CFL criteria: $\Delta t_{f}=4 \times 10^{-5} \mathrm{~s}$.

The sphere is launched at the coordinates of $\left(0.5 L_{x}, 0.5 L_{y}, 0.75 L_{z}\right)$. A value of 20 for $\beta$ and a 0.5 friction coefficient are used.

The mesh is cartesian and uniform $\Delta_{x}=\Delta_{y}=\Delta_{z}=0.091 \mathrm{~cm}$ (about 2.13 millions of cubic cells).

The results for the 3D settling velocity at different Reynolds values are shown in Fig. 9. We observe clearly that Xper captures well the experimental settling velocity magnitudes of the experiment of Ten Cate et al. [1] between 


\begin{tabular}{lll}
\hline Reynolds & $\rho_{f}$ & $\mu$ \\
\hline 1.5 & 970 & 0.373 \\
4.1 & 965 & 0.212 \\
11.6 & 962 & 0.113 \\
32.3 & 960 & 0.058 \\
\hline
\end{tabular}

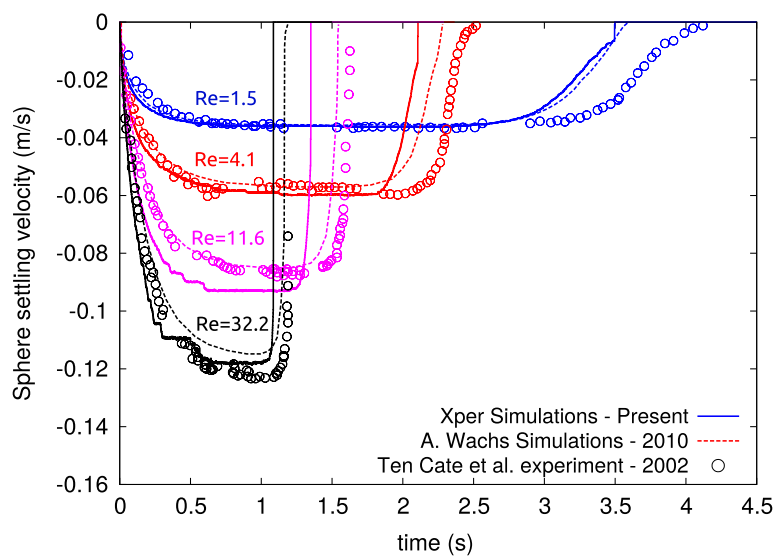

Fig. 9. Sphere settling velocity versus time.

$t=0 \mathrm{~s}$ and $t=4.5 \mathrm{~s}$. These results validate the coupling and the developed Xper platform. Indeed, our results are also in very good agreement with the simulations of Wachs [26] who used a different numerical method (a DEM-DLM/FD method).

However, a difference is observed between the numerical and experimental results near the lower wall. This might be explained either by experimental artifacts when using the Particles Image Velocimetry (PIV) technique near the walls, and/or might be due to the lack of numerical precision in capturing well lower velocity magnitudes near the wall due to very small scale lubrication forces. A fact is that in our work, no lubrication force is modeled when the sphere approaches the bottom wall. However, a detailed study about the effects of lubrication forces can be found in the recent works of Izard et al. [27]. The latter authors introduced the lubrication force modeling into the discrete element method, which is not captured usually by the fluid solver at a very small scale. This effect of lubrication forces near the wall will be addressed in a precise study in the future using our developed solvers in Xper platform where a very fine mesh near the lower wall will be mandatory.

\section{Application: effect of the fluid ring on the settling of two cylinders initially aligned vertically}

In the coming sections, we mean by the term "fluid-ring" any fluid mesh cells that are imposed numerically around mesh cells representing a solid region. When it comes to SSI, most numerical methods in the literature impose a numerical fluid ring around the immersed solids to avoid numerical convergence difficulties. However, this fluid ring cannot represent the real cases. In that manner, the developed platform Xper is used here to compute contacts dynamics problem of two immersed rigid cylinders in an attempt to clarify this important issue and to emphasize the effect of imposing a very small numerical fluid ring on changing the dynamics between solids after contacts.

The application deals with a 2D settling of two cylinders initially aligned vertically.

The domain is rectangular with $L=0.32 \mathrm{~cm}$ and $H=3.2 \mathrm{~cm}$. The cylinders are of $D=2 R=0.08 \mathrm{~cm}$ with a density $\rho_{s}=1200 \mathrm{~kg} / \mathrm{m}^{3}$. The parameters of the fluid are $\mu=0.004313 \mathrm{~Pa} \mathrm{~s}$ and $\rho_{f}=1000 \mathrm{~kg} / \mathrm{m}^{3}$.

A uniform mesh is used with $\left(\Delta_{x}=\Delta_{y}=D / 20\right)$ and the time step $\Delta t_{f}=10^{-5} \mathrm{~s}$ insures a $C F L \ll 1$. A value of 20 for $\beta$ and 0.5 friction coefficient is used. 


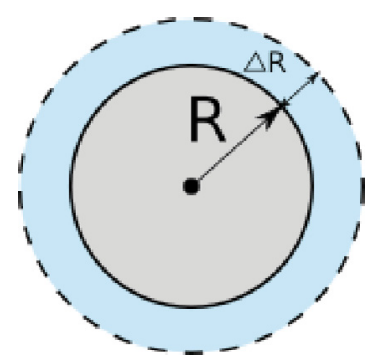

Fig. 10. Rigid cylinder with an imposed numerical fluid ring of $\Delta R$.

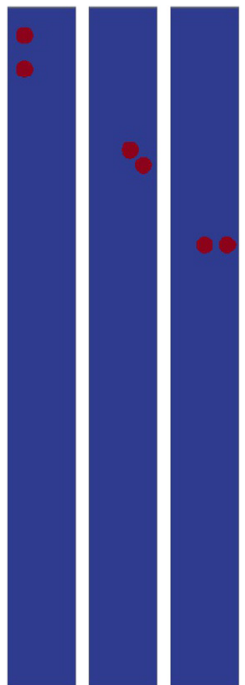

(a) With fluid ring.
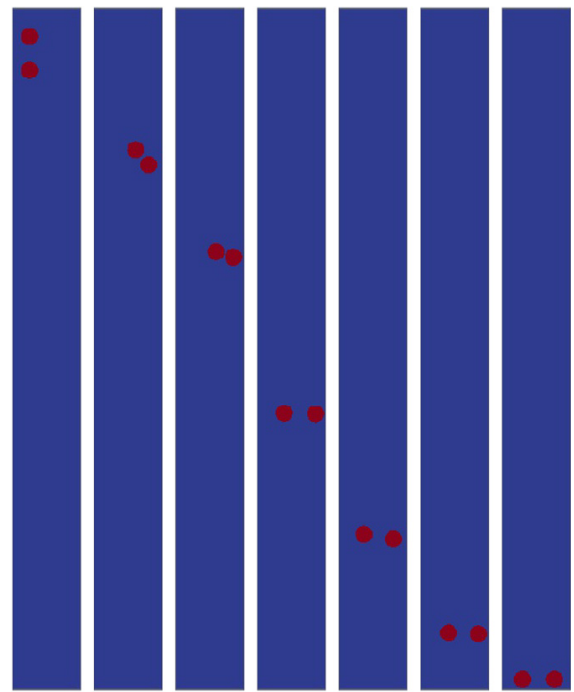

(b) Without fluid ring.

Fig. 11. 2D settling of two cylinders initially aligned vertically and off-centerline at $t=0 \mathrm{~s}, t=0.5 \mathrm{~s}, t=1 \mathrm{~s}, t=2.5 \mathrm{~s}, t=4 \mathrm{~s}, t=5 \mathrm{~s}$ and $t=6.5 \mathrm{~s}$.

Fig. 10 shows a rigid cylinder with a fluid ring of $\Delta R$ thickness. Two cases are tested to study the effect of friction on the contacts dynamics between the two cylinders: $\Delta R=0.1 R$ where friction is absent and $\Delta R=0$ where friction is present.

In each case, we investigate two initial alignment off-centerline and on-centerline.

\subsection{Off-centerline}

The two cylinders are initially aligned vertically off-centerline at the following centers: $(0.25 \mathrm{~L}, 0.96 \mathrm{H})$ and $(0.25 \mathrm{~L}$, $0.96 \mathrm{H}-2 \mathrm{D})$.

Fig. 11 shows snapshots of the particle motion at different time. The principal interactions between neighboring particles are described as the well-known phenomenon of drafting $(t=0 \mathrm{~s})$, kissing $(t=0.5 \mathrm{~s})$ and tumbling $(t>2.5 \mathrm{~s})$. First, the trailing particle approaches the leading one ("drafting"). After this, the two particles get into contact at approximately $t=0.5 \mathrm{~s}$ ("kissing"). At this time, the gap between the particles is equal to zero without fluid ring or is equal to $\Delta R$ with fluid ring. Following the kissing regime, the particles turn to the horizontal position and fall ("tumbling") at $t>2.5 \mathrm{~s}$ (for more details, see [28]).

Fig. 12 presents the $x$ and $y$ positions of the two cylinders that were initially aligned vertically off-centerline. The dynamics behaviors obtained by imposing a fluid ring and without fluid ring are small and similar. 


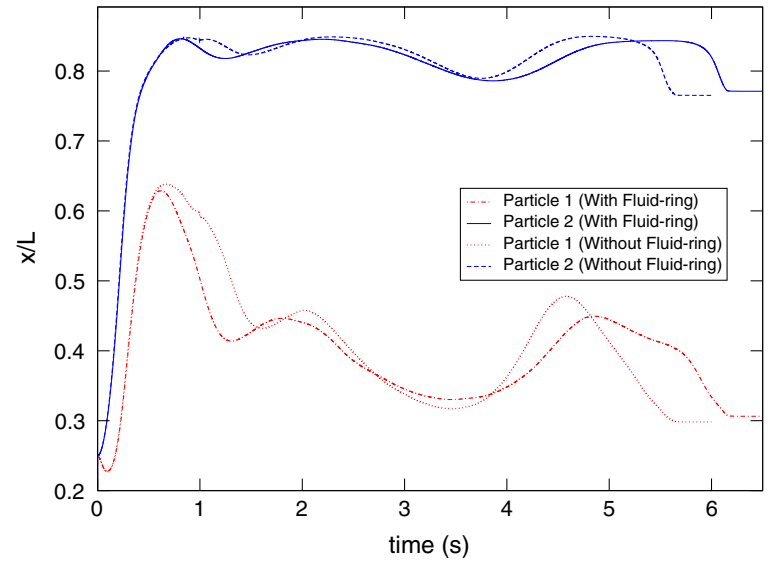

(a) Normalized $x$-positions versus time.

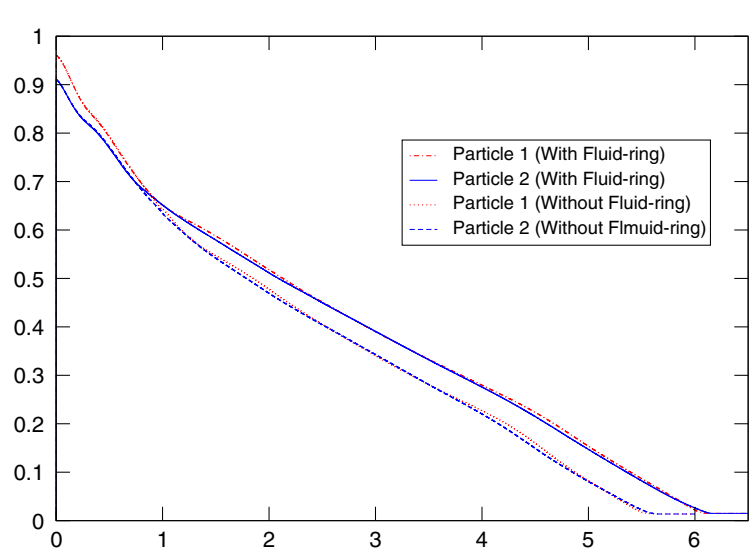

(b) Normalized $y$-positions versus time.

Fig. 12. 2D settling of two cylinders initially aligned vertically and off-centerline.

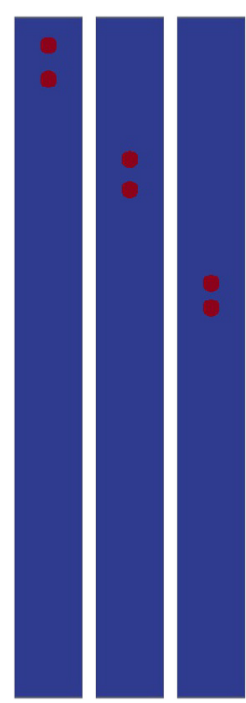

(a) With fluid ring.
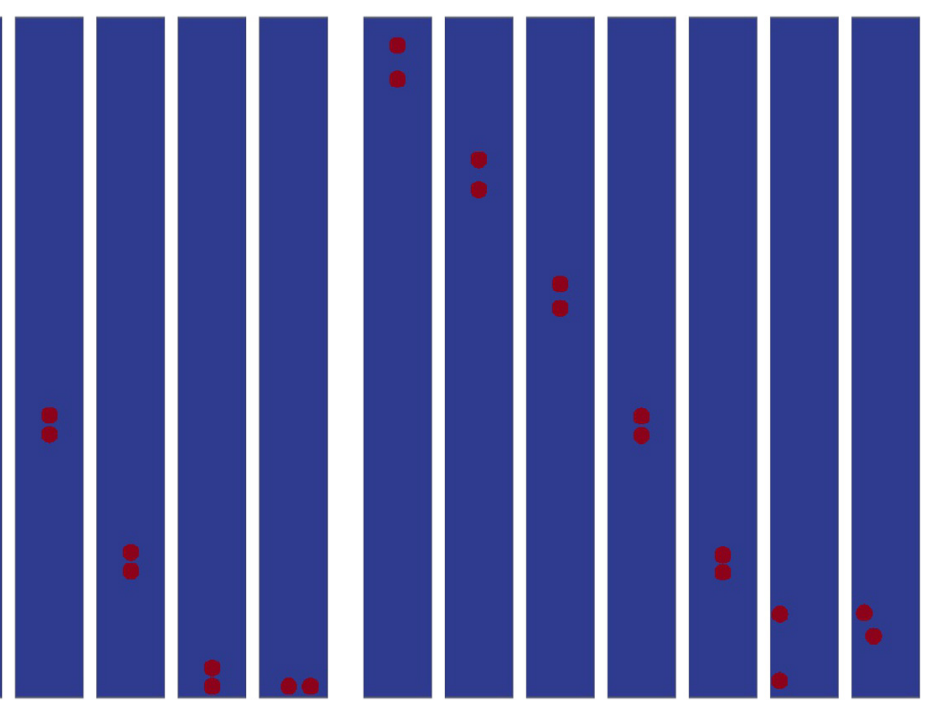

(b) Without fluid ring.

Fig. 13. 2D settling of two cylinders initially aligned vertically and on-centerline at $t=0 \mathrm{~s}, t=0.5 \mathrm{~s}, t=1 \mathrm{~s}, t=1.5 \mathrm{~s}, t=2 \mathrm{~s}, t=2.5 \mathrm{~s}$ and $t=4 \mathrm{~s}$.

\subsection{On-centerline}

The two cylinders are now initially aligned vertically on-centerline at the following centers: $(0.5 \mathrm{~L}, 0.96 \mathrm{H})$ and $(0.5 \mathrm{~L}, 0.96 \mathrm{H}-2 \mathrm{D})$.

Fig. 13 shows snapshots of the particle motion at different time. In this case, only the drafting mechanism is involved. The behaviors are different since the first particle appears to touch the down wall. With friction, i.e. without fluid ring, the particles are pushed away from the down wall. With fluid ring, the particles are sucked all the way to the down wall.

Fig. 14 presents the $x$ and $y$ positions of the two cylinders that were initially aligned vertically on-centerline. We observe a greater effect on the dynamics behavior by imposing a fluid ring, compared to the cases when the two cylinders were initially aligned vertically off-centerline.

The interaction of the particles settling in tandem can be fundamentally altered due to the presence of the fluid ring. The Figs. 12 and 14 emphasize an important new finding. This finding implies that imposing at all even a very small fluid ring, the obtained dynamics cannot correspond well to the "real" phenomena. 


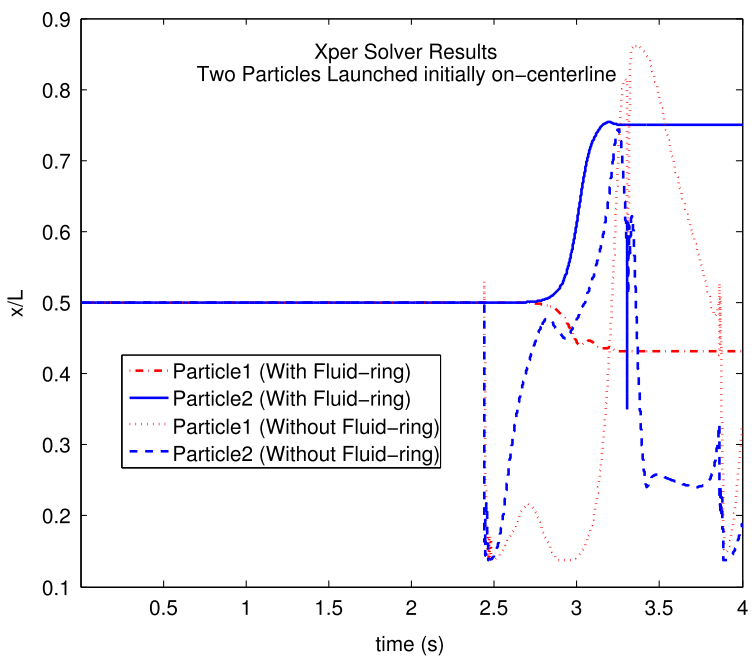

(a) Normalized $x$-positions versus time.

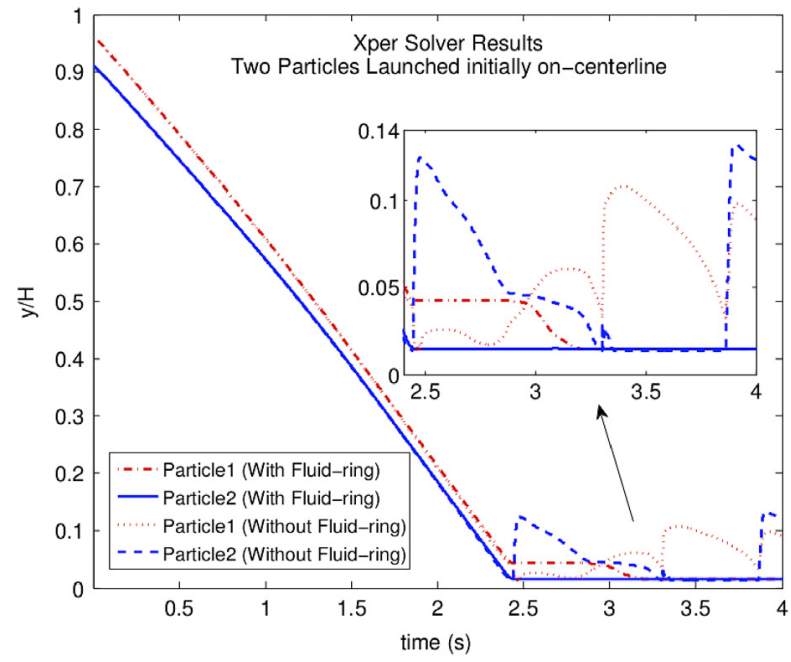

(b) Normalized $y$-positions versus time.

Fig. 14. 2D settling of two cylinders initially aligned vertically and on-centerline.

\section{Conclusion}

A Direct-Forcing Immersed Boundary Method (DF-IBM) is used and implemented in the parallelized numerical framework named PELICANS. This was to take into account for the presence of stationary and moving rigid bodies that are immersed in a fluid (Fluid-Structure Interactions). The numerical implementation is validated by simulating the transient 2D flow around a stationary cylinder at two different Reynold's numbers (20 and 100). Good results are obtained in computing the drag and lift coefficients, and the pressure difference using a PTIT (Penalization Term Integration Technique) inside the immersed body. These results have been characterized by studying the effect of the diameter-to-space-step ratio (DSS) on numerical precision. We found that a DSS of 20 was fair to obtain results that are close to the benchmark data provided by Turek and Schäfer [18] for the 2D flow over a stationary cylinder.

For multiple contacts (Solid-Solid Interactions) between immersed rigid bodies, the PELICANS framework was coupled to the Contact-Mechanics framework named LMGC90 and the resulting package is named Xper.

The 3D settling of a sphere has been simulated at different Reynolds numbers (between 1.5 and 32.2) using Xper to test the validity of the numerical package as a whole. Satisfactory results are obtained for the settling velocity values compared to the experimental and numerical data found in the literature [26,1]. This indicates the primary validity of Xper in simulating immersed granular flows in 3D.

Finally, the effect on the contacts dynamics is addressed by imposing numerical fluid cells around two identical settling cylindrical particles in a stationary fluid as it is usually done by many authors in literature. We found that, imposing this numerical fluid ring (of thickness of the order of a one mesh space) around the particle (as done usually by most authors using different numerical methods to avoid numerical difficulties) to neglect friction alters the particles trajectories. This is a new finding that implies that imposing at all even a very small fluid ring, the obtained dynamics cannot correspond well to the "real" phenomena because the friction due to direct solid-to-solid contacts is present. Moreover, a Coulomb's friction coefficient of 0.5 has shown to have a great effect on the particles dynamics after contact.

This can be crucial in defining the macroscopic behavior when more than two particles may enter in contact during an immersed granular flow based on a certain microstructure orientation.

Further work is required to study the effect of introducing a level-set method, i.e. a smooth edge for the Fluid-Solid Interface, on the numerical results of the DF-IBM and on the computation time performance. Further work is also required to study the effect of a range of Coulomb's friction coefficient values on the particles dynamics.

Numerical simulations involving immersed granular flows, i.e. thousands of particles, will be conducted in the near future using Xper in an attempt to constitute macroscopic rheology laws taking into account the physics at the microscopic scale. 


\section{Acknowledgments}

The author appreciated and thanks V. Topin, F. Dubois, J.-C. Latché and B. Piar for their useful scientific discussions.

\section{References}

[1] A. Ten Cate, C.H. Nieuwstad, J.J. Derksen, H.E.a. Van den Akker, Particle imaging velocimetry experiments and lattice-Boltzmann simulations on a single sphere settling under gravity, Phys. Fluids 14 (11) (2002) 4012. http://dx.doi.org/10.1063/1.1512918.

[2] N. Blaise, The dam break problem for a hyperconcentrated suspension, Appl. Rheol. 10 (5) (2000) 224-230.

[3] F. Blanc, E. Lemaire, F. Peters, Tunable fall velocity of a dense ball in oscillatory cross-sheared concentrated suspensions, J. Fluid Mech. 746 (2014) 1-10. http://dx.doi.org/10.1017/jfm.2014.160.

[4] C. Peskin, Flow patterns around heart valves: A numerical method, J. Comput. Phys. 10 (2) (1972) 252-271.

[5] C. Peskin, Numerical analysis of blood flow in the heart, J. Comput. Phys. 25 (1977) 220-252.

[6] C. Peskin, The fluid dynamics of heart valves: Experimental, theoretical, and computational method, Annu. Rev. Fluid Mech. 14 (1982) $235-259$.

[7] J. Mohd-Yusof, Combined immersed-boundary/b-spline methods for simulations of flow in complex geometries, in: S. University (Ed.), Annual Research Briefs, Center for Turbulence Research, Stanford, CA, 1997, pp. 317-327.

[8] E. Fadlun, R. Verzicco, J. Mohd-Yusof, Combined immersed boundary finite difference methods for three-dimensional complex flow simulations, J. Comput. Phys. 161 (1) (2000) 35-60.

[9] R. Mittal, G. Iaccarino, Immersed boundary methods, Annu. Rev. Fluid Mech. 37 (2005) 239-261. http://dx.doi.org/10.1146/annurev.fluid.37.061903.175743.

[10] F.H. Harlow, J.E. Welch, Numerical calculation of time-dependent viscous incompressible flow of fluid with free surface, Phys. Fluids 8 (12) (1965) 2182-2189.

[11] A.J. Chorin, Numerical solution of the Navier-Stockes equations, Math. Comp. 22 (104) (1968) 745-762.

[12] J. Van Kan, A second-order accurate pressure-correction scheme for viscous incompressible flow, SIAM J. Sci. Stat. Comput. 7 (1986) 870.

[13] S. Turek, Efficient Solvers for Incompressible Flow Problems: An Algorithmic Approach in View of Computational Aspects, 1998.

[14] J.-p. Caltagirone, Sur I'interaction fluide-milieu poreux; application au calcul des efforts exerces sur un obstacle par un fluide visqueux, C. R. Acad. Sci. 318 (II) (1994) 571-577.

[15] K. Khadra, P. Angot, S. Parneix, J.-p. Caltagirone, Fictitious domain approach for numerical modelling of Navier - Stokes equations, Internat. J. Numer. Methods Fluids 34 (2000) 651-684.

[16] T. Zohdi, Embedded electromagnetically sensitive particle motion in functionalized fluids, Comput. Part. Mech. 1 (2014) $27-45$.

[17] https://gforge.irsn.fr/gf/project/pelicans.

[18] M. Schäfer, S. Turek, Benchmark computations of laminar flow around a cylinder, in: E. Hirschel (Ed.), Flow Simulation with HighPerformance Computers II, in: Notes on Numerical Fluid Mechanics, vol. 52, 1996.

[19] J. Oden, E. Pires, Nonlocal and nonlinear friction laws and variational principles for contact problems in elasticity, ASME J. Appl. Mech. 50 (1983) 67-76.

[20] J. Martins, J. Oden, Existence and uniqueness results in dynamics contact problems with nonlinear normal and friction interfaces, Nonlinear Anal. 11 (1987) 407-428.

[21] M. Jean, The non-smooth contact dynamics method, Comput. Methods Appl. Mech. Engrg. 177 (1999) 235-257.

[22] https://git-xen.lmgc.univ-montp2.fr/lmgc90/.

[23] J.-J. Moreau, Unilateral contact and dry friction in finite freedom dynamics, in: J.-J. Moreau, P. Panagiotopoulos (Eds.), Non Smooth Mechanics and Applications, in: CISM-Courses and Lectures, vol. 302, Springer, Vienna, 1988, pp. 1-82.

[24] T. Zohdi, Computation of strongly coupled multifield interaction in particle-fluid systems, Comput. Methods Appl. Mech. Engrg. 196 (2007) 3927-3950.

[25] T. Zohdi, Simulation of coupled microscale multiphysical-fields in particulate-doped dielectrics with staggered adaptive fdtd, Comput. Methods Appl. Mech. Engrg. 199 (2010) 79-101.

[26] A. Wachs, Peligriff, a parallel dem-dlm/fd direct numerical simulation tool for 3d particulate flows, J. Eng. Math. 71 (1) (2011) 131-155.

[27] E. Izard, T. Bonometti, L. Lacaze, Modelling the dynamics of a sphere approaching and bouncing on a wall in a viscous fluid, J. Fluid Mech. 747 (2014) 422-446.

[28] S. Ghosh, J.M. Stockie, Numerical simulations of particle sedimentation using the immersed boundary method, ArXiv e-prints (2013) 1-46. 